\title{
Urinary Proteomics Evaluation in Interstitial Cystitis/Painful Bladder Syndrome: A Pilot Study
}

\author{
Young Ah Goo, Yihsuan S. Tsai, Alvin Y. Liu, David R. Goodlett, Claire C. Yang \\ Department of Medicinal Chemistry (YAG, YST, DRG), Department of Urology (AYL, CCY), and In- \\ stitute for Stem Cell and Regenerative Medicine (AYL), University of Washington, Seattle, WA, USA, \\ Institute for Systems Biology (DRG), Veterans Affairs Puget Sound Health Care System (CCY), Seattle, \\ $W A, U S A$
}

\begin{abstract}
Purpose: Interstitial cystitis/painful bladder syndrome (IC/PBS) is characterized by chronic pain, pressure and discomfort felt in the pelvis or bladder. An in-depth shotgun proteomics study was carried out to profile the urinary proteome of women with IC/PBS to identify possible specific proteins and networks associated with IC/PBS.

Materials and Methods: Urine samples from ten female IC/PBS patients and ten female asymptomatic, healthy control subjects were analyzed in quadruplicate by liquid chromatography-tandem mass spectrometry (LC-MS/MS) on a hybrid linear ion trap-orbitrap mass spectrometer. Gas-phase fractionation (GPF) was used to enhance protein identification. Differences in protein quantity were determined by peptide spectral counting.

Results: $\alpha$-1B-glycoprotein (A1BG) and orosomucoid-1 (ORM1) were detected in all IC/PBS patients, and $\geq 60 \%$ of these patients had elevated expression of these two proteins compared to control subjects. Transthyretin (TTR) and hemopexin (HPX) were detected in all control individuals, but $\geq 60 \%$ of the IC/PBS patients had decreased expression levels of these two proteins. Enrichment functional analysis showed cell adhesion and response to stimuli were down-regulated whereas response to inflammation, wounding, and tissue degradation were up-regulated in IC/PBS. Activation of neurophysiological processes in synaptic inhibition, and lack of DNA damage repair may also be key components of IC/PBS.

Conclusion: There are qualitative and quantitative differences between the urinary proteomes of women with and without IC/PBS. We identified a number of proteins as well as pathways/networks that might contribute to the pathology of IC/PBS or result from perturbations induced by this condition.
\end{abstract}

Key words: interstitial cystitis; painful bladder syndrome; urine proteomics

Int Braz J Urol. 2010; 36: 464-79

\section{INTRODUCTION}

Interstitial cystitis/painful bladder syndrome (IC/PBS) is defined by chronic pain, pressure and discomfort felt in the lower pelvis or bladder, which are unrelated to any identifiable cause. Urinary urgency and frequency are also common symptoms of IC/PBS. Despite years of intense research, the underlying etiology, pathophysiology, and risk factors for developing and perpetuating this syndrome remain unclear. Diagnosis is based on symptoms and exclusion of other conditions, due to the lack of characteristic pathological findings, well-defined disease phenotypes, or objective biomarkers. Because of 
these barriers, the diagnosis of IC/PBS is frequently delayed, and treatment frequently requires a multimodal approach (1).

One of the hypotheses proposed for the pathophysiology of IC/PBS is disruption of the urothelial barrier leading to symptoms. Bladder surface mucus, composed of glycosaminoglycans (GAGs) and proteoglycans, creates a highly impermeable barrier that is a key to maintain bladder function. Destruction of this barrier leads to tissue infiltration of urinary solutes, in particular potassium, which depolarizes nerves and muscles and causes tissue injury (2). Neurogenic inflammation has also been proposed as a pathophysiologic mechanism in IC/PBS (3). In response to stimuli, urothelial cells could activate neural circuits, releasing factors that cause chronic pain. Both hypotheses could conceivably result in urinary protein byproducts, which could then potentially serve as biomarkers for IC/PBS.

The lack of biomarkers that can be used for diagnosis of IC/PBS or to track treatment efficacy contributes to the clinical burden. Thus, identification of biomarker(s) for IC/PBS would represent a major advance in the field. Cataloging biomolecules present in complex biological samples has become increasingly important in clinical research for the purpose of identifying disease specific biomarkers. In the case of proteins, proteomics uses mass spectrometry to qualitatively and quantitatively catalog proteins. Application of proteomics to human diseases is challenging because about 35,000 human genes could translate into over $1,000,000$ functional protein entities due to post-translational modifications as well as sequence variations (4). In spite of these complexities urinary biomarker discovery holds considerable promise because it has been recently shown that the urinary proteome contains approximately 1,500 proteins (5). This makes the urinary proteome far less complex than the blood proteome where biomarkers are also being sought for various human diseases (4).

In this pilot study, we applied proteomic strategies and related methodologies to profile the urinary proteome of patients with IC/PBS. The potential benefits of this study include a greater understanding of possible causes and underlying mechanisms of IC/PBS.

\section{MATERIALS AND METHODS}

\section{Urine Sample Collection and Processing}

Human urine acquisition was carried out with our institution's Ethics Committee approval. Ten women with a clinical diagnosis of IC/PBS, being treated and followed in the Female Urology Clinic, were enrolled in the study. All women had symptoms of IC/PBS for at least one year, and all had undergone extensive evaluation to exclude reversible, identifiable causes for their pelvic pain and urinary symptoms. Control urine was obtained from ten asymptomatic, pain-free, healthy female subjects, age-matched to the IC/PBS group. One protease inhibitor cocktail tablet (Roche, Indianapolis, IN) was added per $50 \mathrm{~mL}$ urine to avoid proteolysis after urine collection. The urine was centrifuged at $2,000 \mathrm{xg}$ for $10 \mathrm{~min}$ at $4{ }^{\circ} \mathrm{C}$ to remove cells and debris. The supernatant was collected and processed for protein purification by TCA (trichloroacetic acid) precipitation $(10 \% \mathrm{w} / \mathrm{v})$. Protein concentration was measured by $\mathrm{BCA}^{\mathrm{TM}}$ protein assay (Thermo Fisher, Waltham, MA). Proteins, 200 $\mu$ g each per subject, were reduced, alkylated, digested with trypsin (Promega, Madison, WI), and then desalted.

\section{Mass Spectrometry Analysis}

Peptide digests were analyzed by electrospray ionization on a hybrid linear ion trap-orbitrap mass spectrometer (Thermo Fisher). For each liquid chromatography-tandem mass spectrometry (LC-MS/MS) analysis, approximately $0.5 \mu \mathrm{g}$ of peptides were loaded on the column and eluted in acetonitrile gradient (6). To maximize protein identification without protein fractionation, ions were selected via a data-dependent process from 400-2,000 Th or by gas-phase fractionation (GPF) from 400-521, 516-690, 685-968, and 963-2,000 Th (6). Each experiment was acquired in quadruplicate.

\section{Database Search and Protein Identification}

Acquired tandem mass spectra (MS/MS) were searched for sequence matches against the Interna- 
tional Protein Index (IPI) human protein database using SEQUEST. PeptideProphet and ProteinProphet, which compute a probability likelihood of each identification being correct, were used for statistical analysis (7). Only proteins identified by more than one unique peptide sequence were included in the analysis. Differences in protein expression were calculated using peptide spectral counting algorithms that use MS/MS data to estimate changes in relative abundance of proteins (8).

\section{Western Blot Analysis}

Ten $\mu \mathrm{g}$ of pooled IC/PBS or control urine protein was resolved on $4-12 \% \mathrm{NuPAGE}^{\circledR}$ gel (Invitrogen, Carlsbad, CA) and transferred to PVDF membrane for incubation with primary antibodies, followed by HRP-conjugated secondary antibodies (Amersham, Piscataway, NJ). Reactivity was visualized by enhanced chemiluminescence (Amersham).

\section{RESULTS}

\section{IC/PBS and Control Urinary Proteomes}

GPF increased protein identification by more than $60 \%$ over the use of one large $\mathrm{m} / \mathrm{z}$ range in both sets of urine samples (Figure-1A). A total of 889 IC/PBS and 1003 control proteins with Protein Probability $\geq 0.8$, with error rates $\leq 0.023$ and $\leq 0.022$ respectively, were identified. Recently, the normal urine proteome was extensively analyzed revealing more than 1,500 proteins (5). A comparative analysis of our IC/PBS and control urines, and normal urine data by Adachi et al. (5) is shown in a Venn diagram (Figure-1B) created by ProteinCenter (www.proxeon . com). According to this analysis, 165 proteins appeared to be unique to IC/PBS. However, proteins identified in only one mutually exclusive subset may be due to under-sampling in other samples or result from data filtering (7).

Identified urine proteins were annotated with Gene Ontology (GO) (9), which assign probable subcellular compartmentalization and molecular functions. Approximately $50 \%$ of the proteins identified were annotated as secreted or membrane-associated proteins, which may be a characteristic of the urine proteome (5).

\section{Proteins Associated with IC/PBS}

A total of 78 proteins with P-value $\leq 0.1$ were considered to be statistically significant for differential expression between IC/PBS and control for this study (Table-1). This P-value was chosen to cast a wider net that includes the most of the differentially expressed proteins. By quantitative analysis, 19 were found up-regulated in IC/PBS compared to control, and 59 were down-regulated. Among these, we focused on proteins identified in all ten IC/PBS subjects and were up-regulated in at least $60 \%$ of this cohort by spectral counts. Similarly, proteins that were found in all ten control subjects, and were down-regulated in more than $60 \%$ of IC/PBS cohort were also investigated. Using these criteria, two upregulated and two down-regulated proteins were found. The two up-regulated were $\alpha$-1B-glycoprotein (A1BG) and orosomucoid-1 (ORM1) both of which are glycoproteins. This is promising in that many current biomarkers like prostate specific antigen (PSA) are glycoproteins (7). A1BG is a plasma glycoprotein of unknown function but over-expression of this protein in pancreatic adenocarcinoma patients has been reported (10). ORM1 is an acute phase plasma protein that increases as a result of acute inflammation (11). The two down-regulated proteins were transthyretin (TTR) and hemopexin (HPX). TTR is a thyroid hormone-binding protein. Defects in TTR are the cause of amyloidosis (12). HPX protects low-density lipoprotein against hemoglobin-induced oxidation (13). Differential expression of these proteins was further validated by Western blot analysis of pooled IC/PBS and control samples (Figure-2). Among the other up-regulated proteins, afamin (APF), osteopontin (SPP1), pancreatic secretory trypsin inhibitor (SPINK1), proactivator polypeptide (PSAP), and apolipoprotein (LPA) were found from all ten IC/PBS patients.

Enrichment functional analysis, which ranks the most relevant cellular processes among the differentially expressed proteins, was performed by MetaCore $^{\mathrm{TM}}$ pathway analysis tool (www.genego.com). Cellular process networks such as inflammatory response, tissue degradation, and wounding response 
A

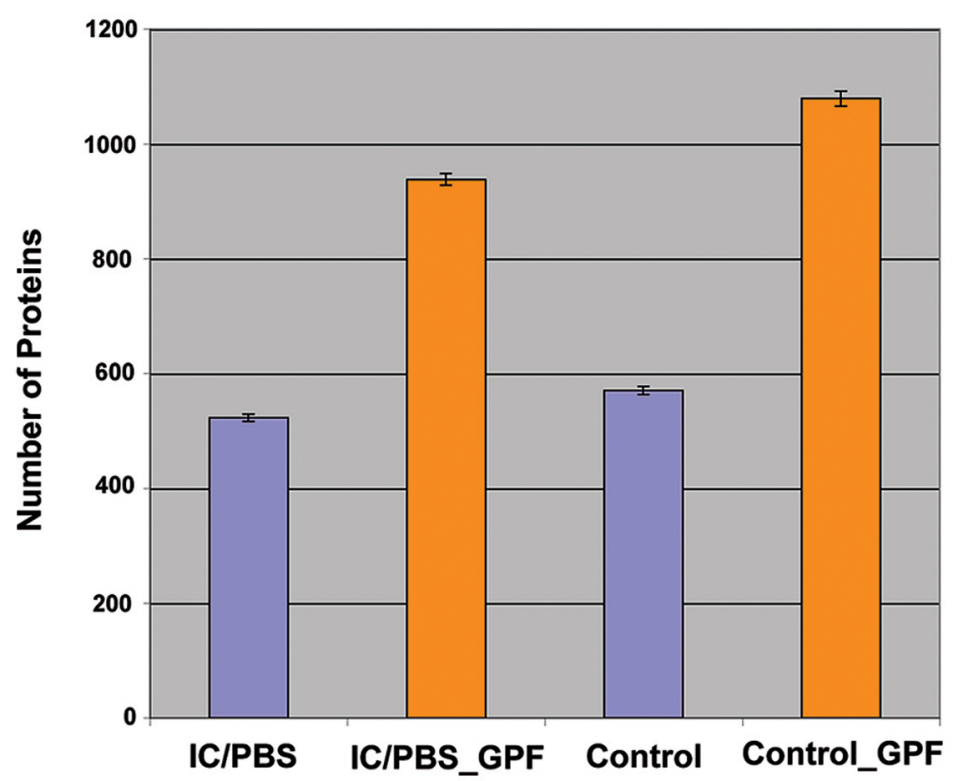

B

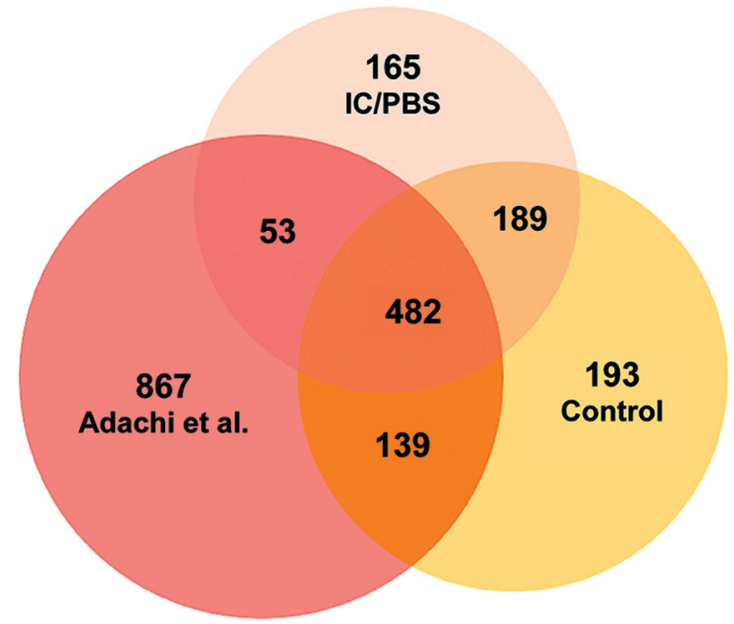

Figure 1 - IC/PBS and control urine protein identifications. (A) Average number \pm standard deviation (on 4 technical replicates) of identified proteins with 2 or more peptides (multiple hits) using single $\mathrm{m} / \mathrm{z}$ or 4 gas-phase $\mathrm{m} / \mathrm{z}$ fractions for IC/PBS and control urine. (B) Our urinary proteome of IC/PBS and controls were compared to the previously reported normal urine proteome (Adachi et al. 2006). The Venn diagram shows overlapped proteins (621) between our asymptomatic control proteins and previously published healthy urine proteins. This comparative analysis also identified 165 proteins that may be unique to the IC/PBS proteome. However, proteins identified in one sample only may be due to under-sampling in other sample or result from data filtering.

were found up-regulated in IC/PBS, whereas cell adhesion, extra cellular matrix remodeling, and stimulus response were found to be down-regulated.

When the 165 IC/PBS proteins (Figure1B) were queried for pathways, neurophysiological GABA-A receptor life cycle pathway was mapped with the most number of proteins. Gamma-amino butyric acid receptors (GABA-A) mediate fast synaptic inhibition in the brain and spinal cord (14). Alterations in neuronal surface receptors modulate the synaptic strength, leading to changes in sensitivity to neurotransmitters (15). When a similar network analysis was performed for the 193 control proteins, 


\section{IC/PBS Control}

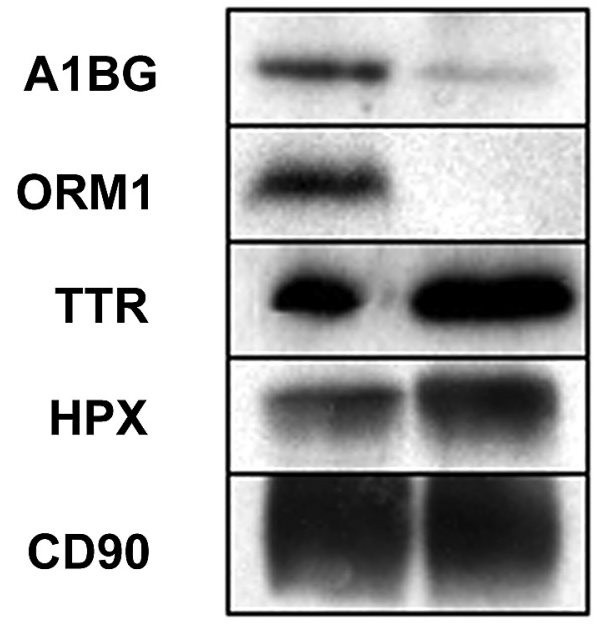

Figure 2 - Western blot analysis of differentially expressed proteins. Ten $\mu \mathrm{g}$ of pooled proteins were resolved by gel electrophoresis and probed by antibodies. The expression level as indicated by the band intensity correlated well with the spectral count quantification method. Shown are the results for $\alpha-1 B$-glycoprotein (A1BG), orosomucoid-1 (ORM1), transthyretin (TTR), and hemopexin (HPX). CD90 (Thy-1) served as the control for sample loading. CD90 is a GPI-anchored protein also found secreted.

DNA damage regulation pathway was one of the most activated pathways, suggesting a lack of DNA damage regulation and repair functions in IC/PBS.

\section{In Silico Analysis of Tissue Specific Expression}

Although the proteins identified in this study are found in urine, some of the proteins identified may be more highly expressed in a specific tissue type, and their tissue specificity could enhance understanding of the mechanisms underlying IC/PBS. Among the differentially expressed proteins, cell adhesion molecule with homology to L1CAM (CHL1) is highly expressed in the cortex, brain, and spinal cord based on UniProt tissue classifications (www.uniprot.org). CHL1 is a neural recognition molecule involved in signal transduction pathways, and loss of this gene is responsible for mental defects (16). In our datasets, CHL1 was down-regulated in IC/PBS.

\section{Protein-protein Interaction Network}

Protein-protein interactions are important in signal transduction, which plays a fundamental role in many biological processes and diseases. The differentially expressed proteins were investigated for novel protein-protein interactions using MetaCore ${ }^{\mathrm{TM}}$. Direct and indirect protein interactions were ranked and interpreted in terms of GO processes. Two novel protein network modules with potential importance in IC/PBS were identified: 1) glucose metabolic process and positive regulation of natural killer cell-mediated immune response to tumor cells, and 2) response to external stimulus, cell adhesion, wounding, and stress.

\section{COMMENTS}

Much effort has been devoted to the search for useful biomarkers for IC/PBS diagnosis, phenotyping, and for predicting response to treatment (17). Initial attempts to develop a urinary biomarker concentrated on mediators of pain such as substance P (18). Other proposed pain biomarkers have included uroplakin III$\delta 4$ mRNA(19), and heparin-binding epidermal growth factor-like growth factor (HB-EGF) (20). Antiproliferative factor (APF) is another candidate (21). To date, none of these has been shown to definitively correlate with IC/PBS symptoms, clinical course, or response to treatment. In a recent urine biomarker evaluation study, no robust association among urinary IL-6, cyclic guanosine monophosphate, HB-EGF, epidermal growth factor, APF, and bladder biopsy was found in IC/PBS (22). Previously, a urinary proteomic method was applied to identify biomarkers from age-, race-, and gender-matched IC/PBS and control subjects (23). Three up-regulated proteins (uromodulin and two kininogens) in the control and one up-regulated protein (inter- $\alpha$-trypsin inhibitor heavy chain $\mathrm{H} 4$ ) in the IC/PBS were found. These proteins showed a correlation to IC severity on IC-specific quality-of-life scales. All four proteins were also found in our study but their differential expressions were not statistically significant in our datasets.

The goal of this study was to use an in-depth proteomic approach to identify specific proteins or 


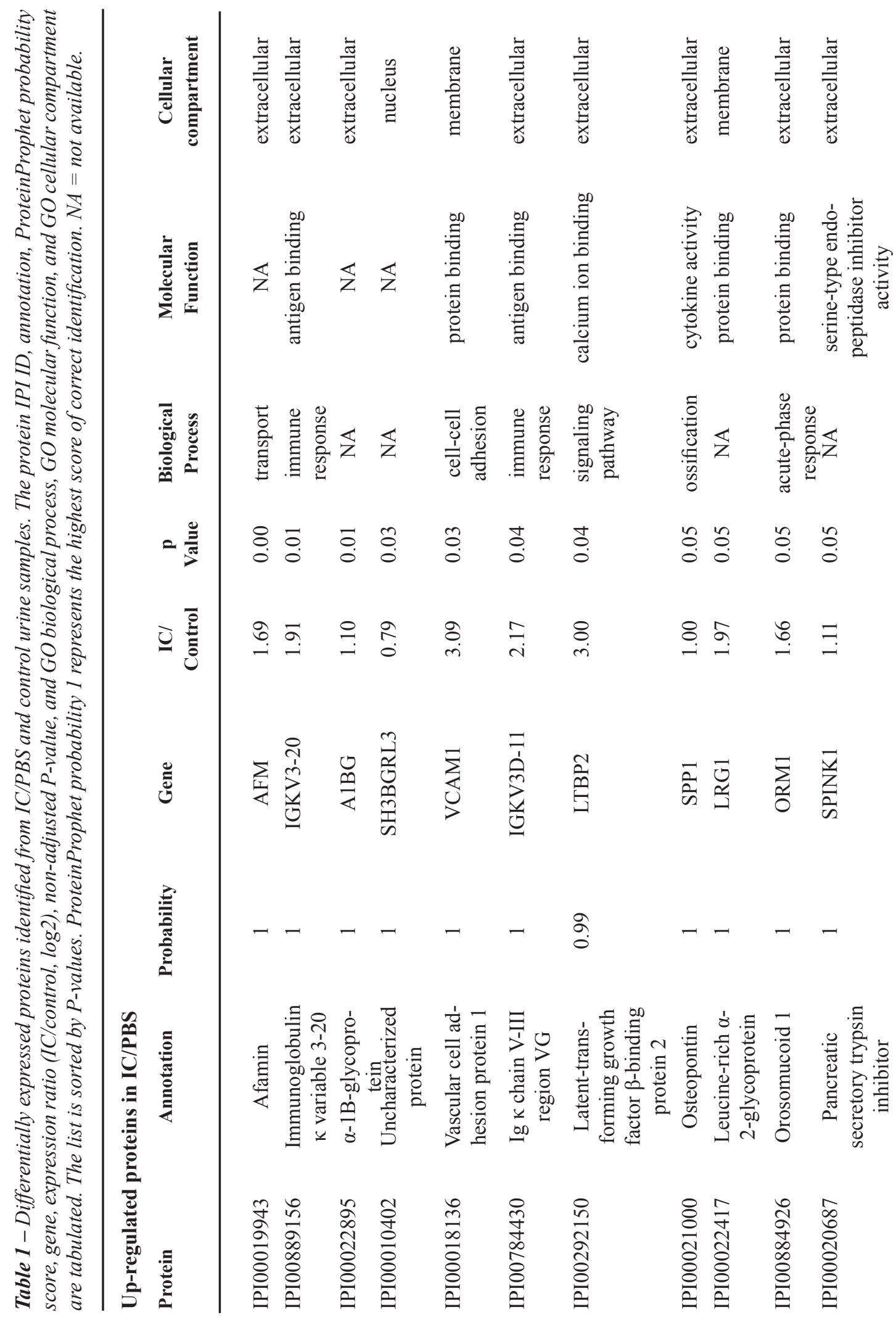




\section{Urinary Proteomics in IC/PBS}
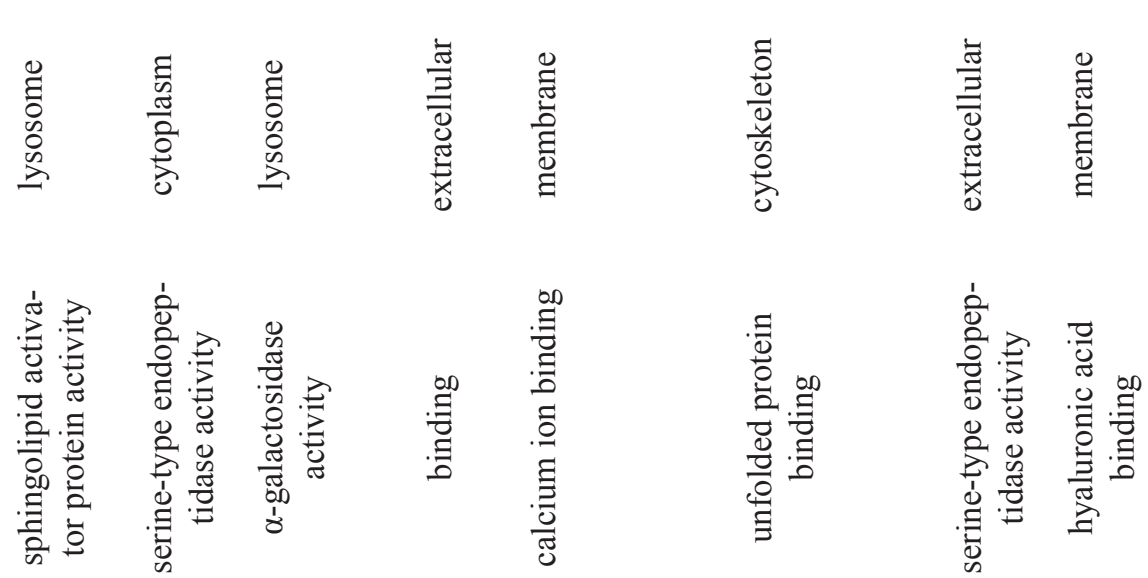

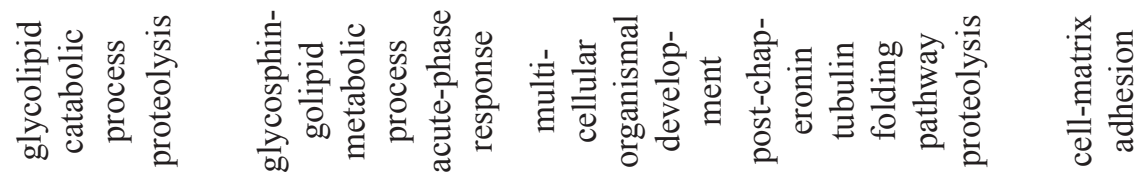

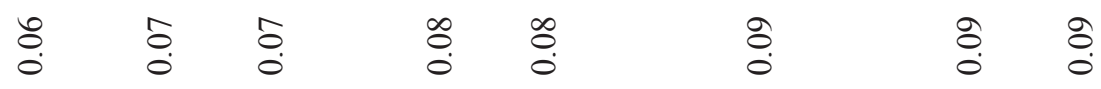

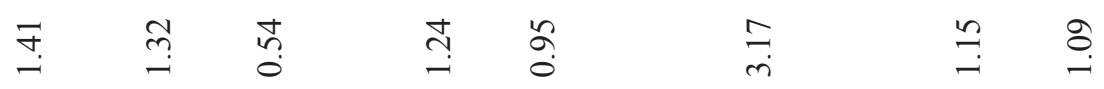

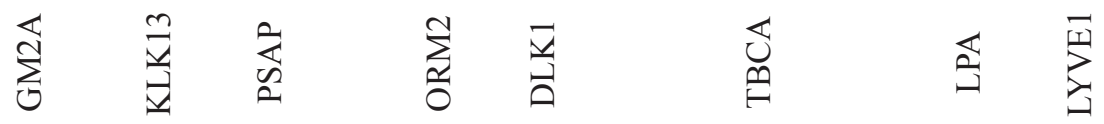

$\begin{array}{llllllll} & 0 & 0 & - & - & - & 0 & -\end{array}$

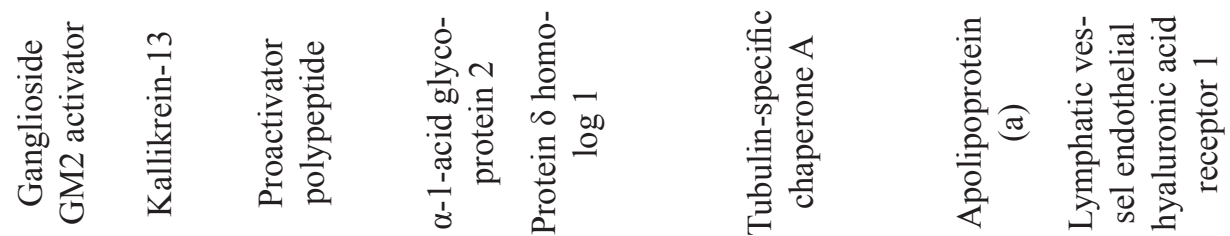

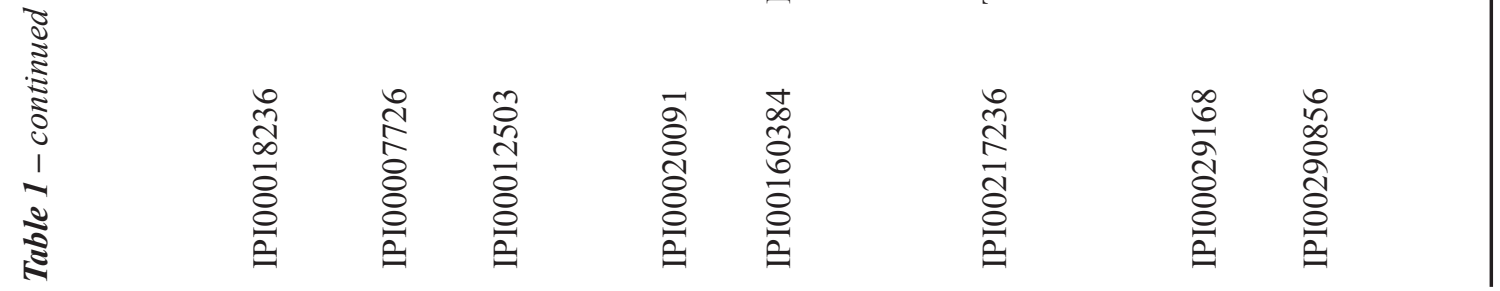




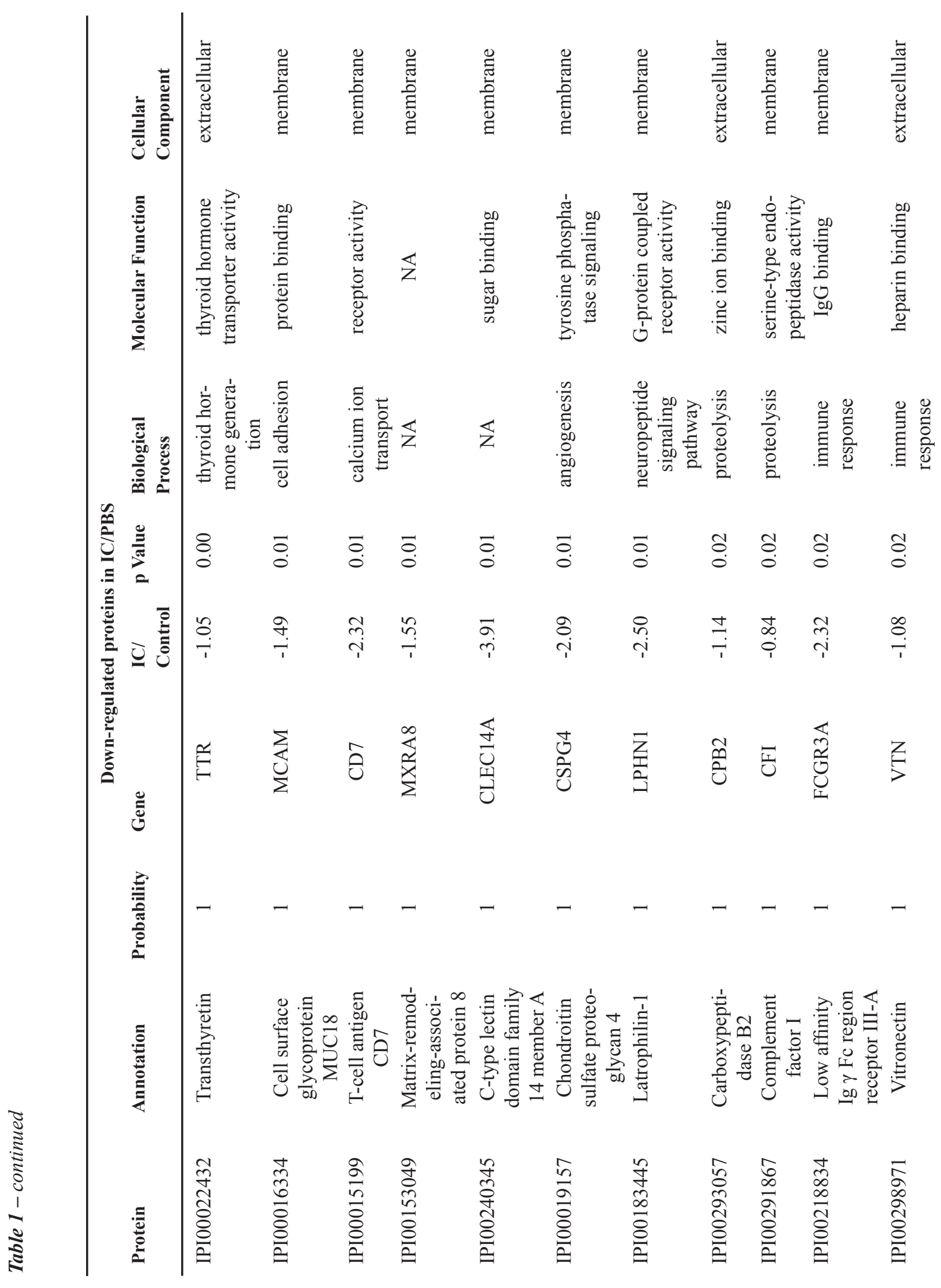




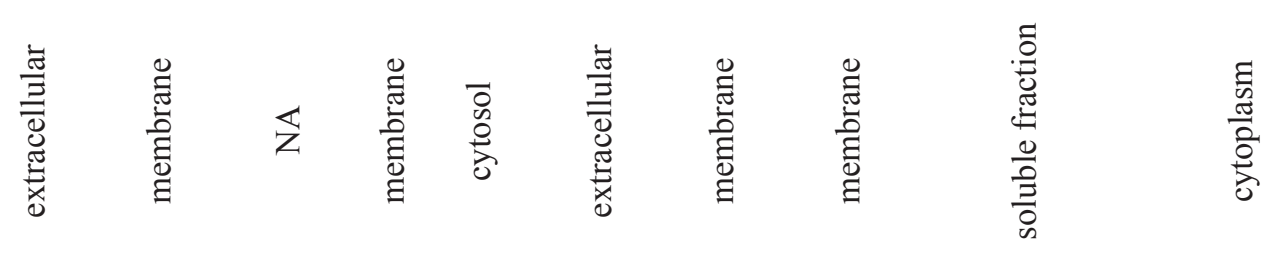

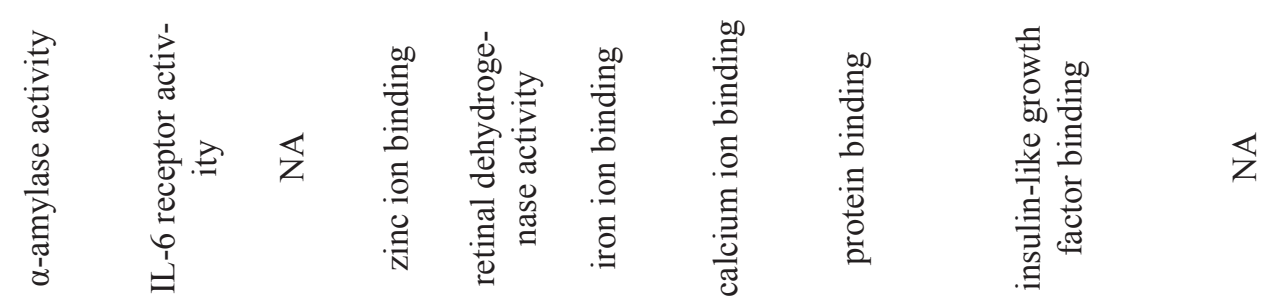

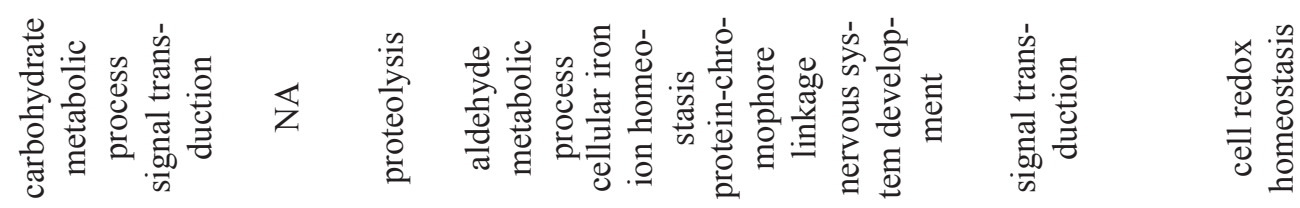

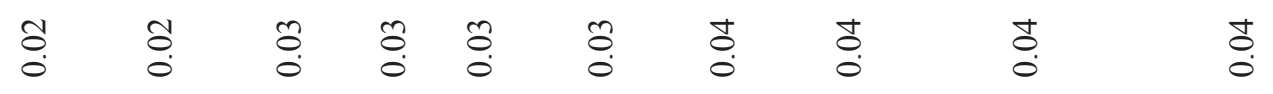

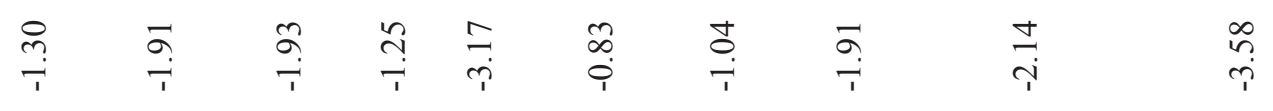

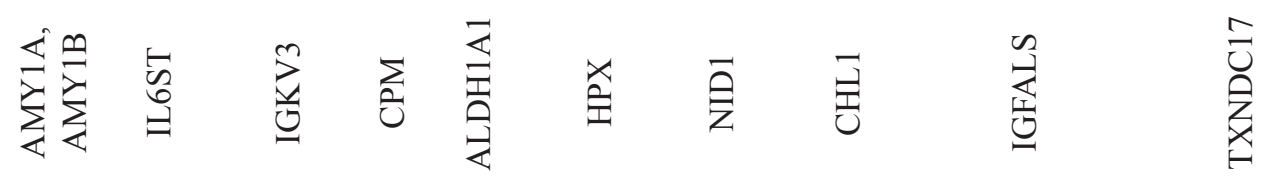

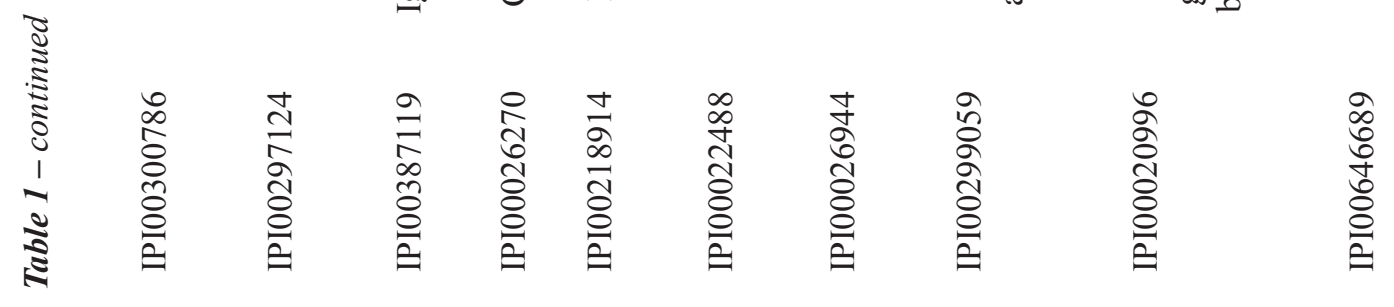

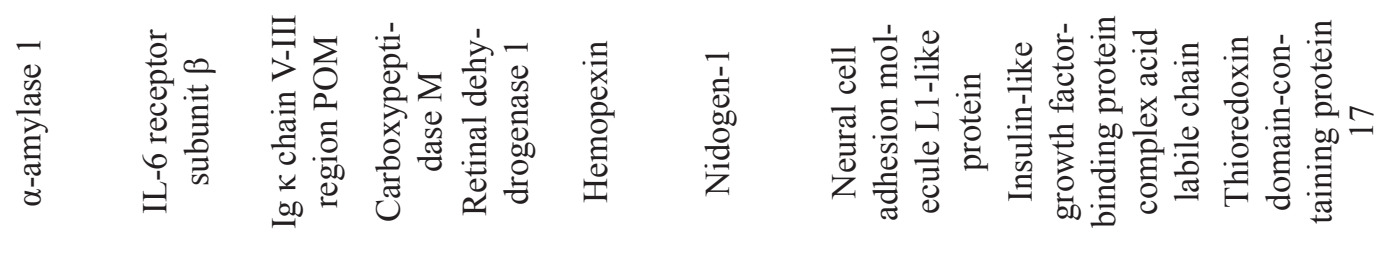




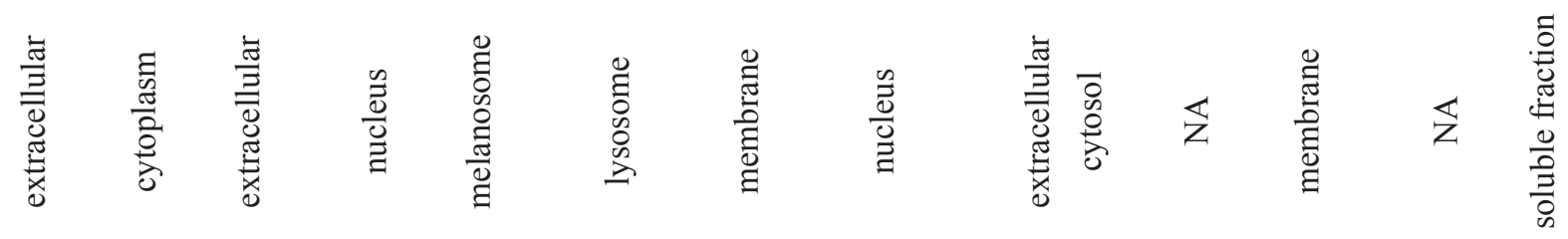

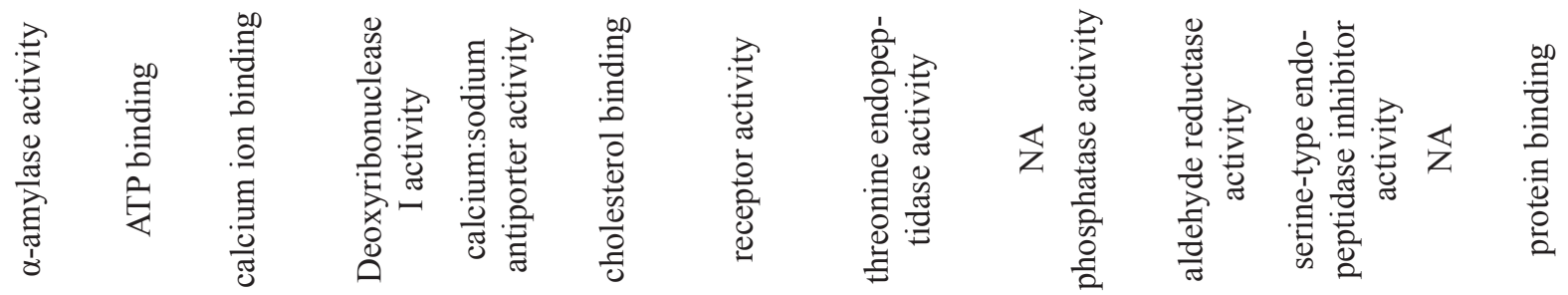

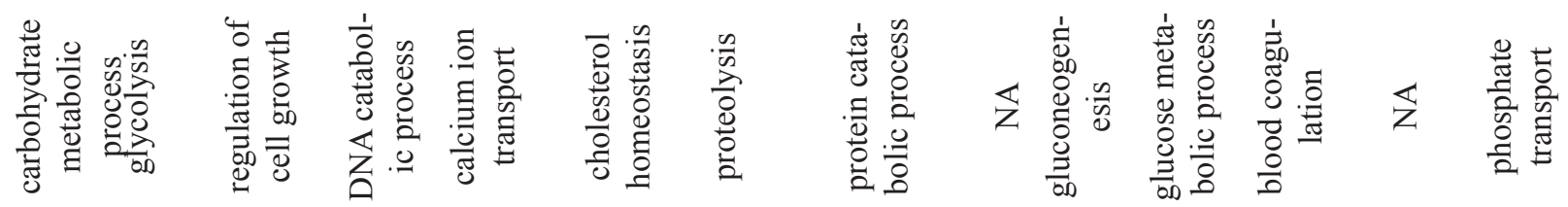
乡ั

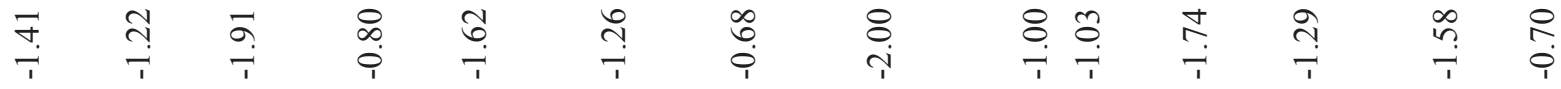

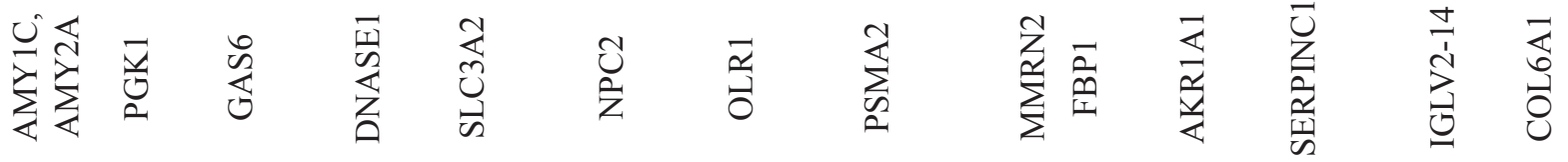

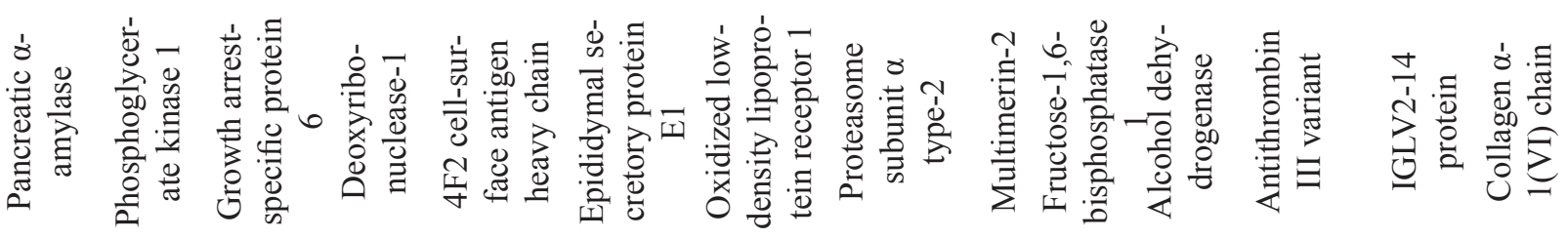




\section{Urinary Proteomics in IC/PBS}

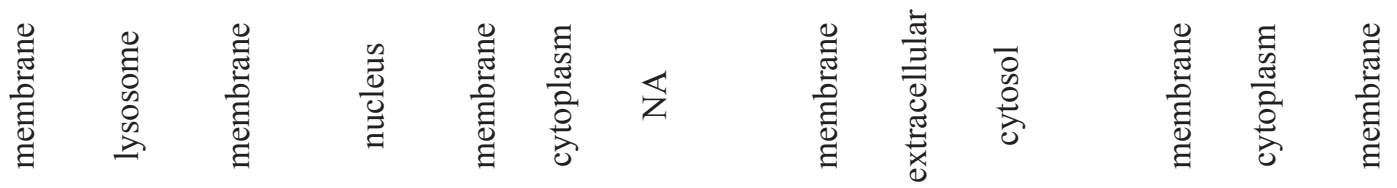

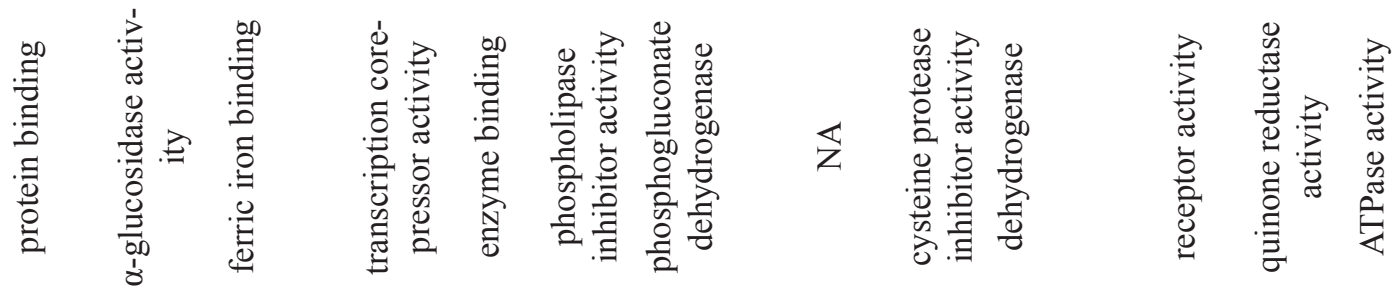

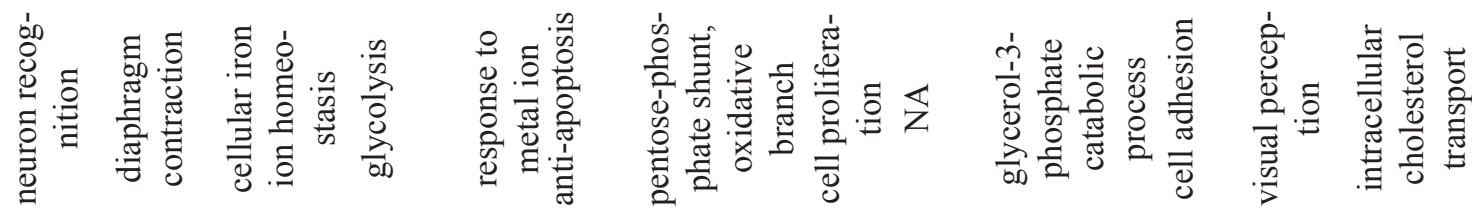

ไ气

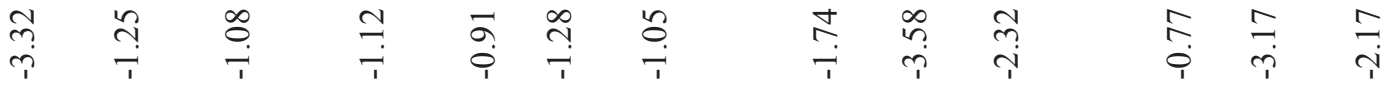

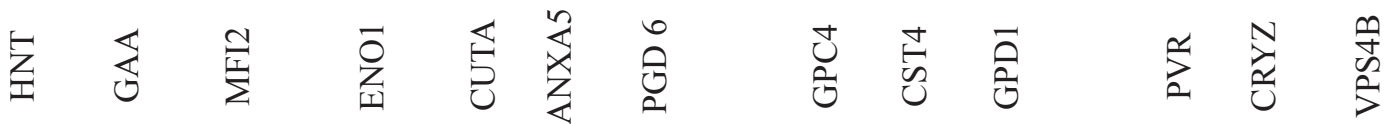

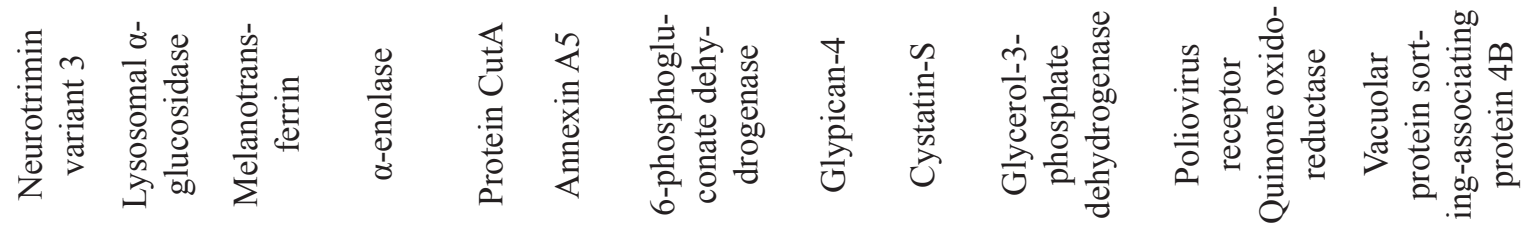

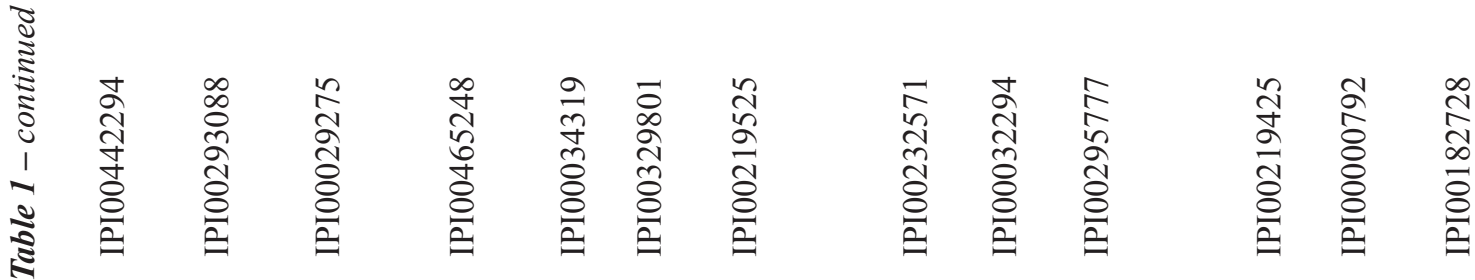




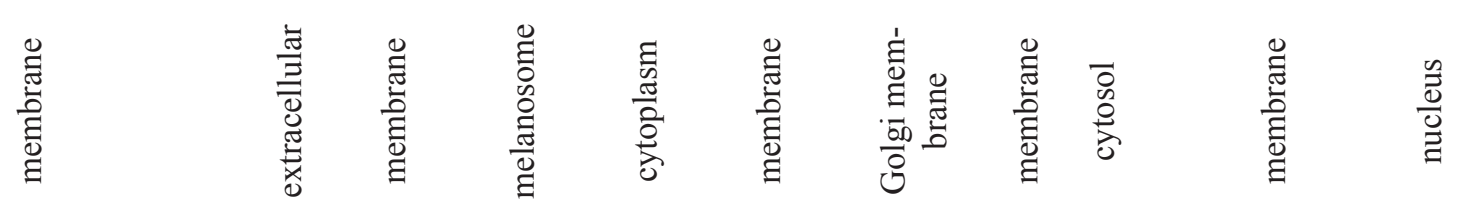

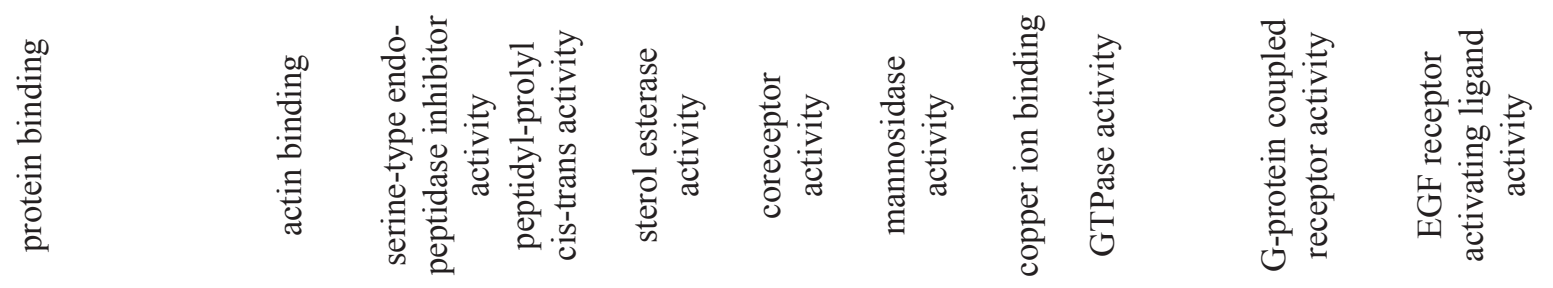

$\frac{\overline{0}}{\sqrt[0]{0}}$

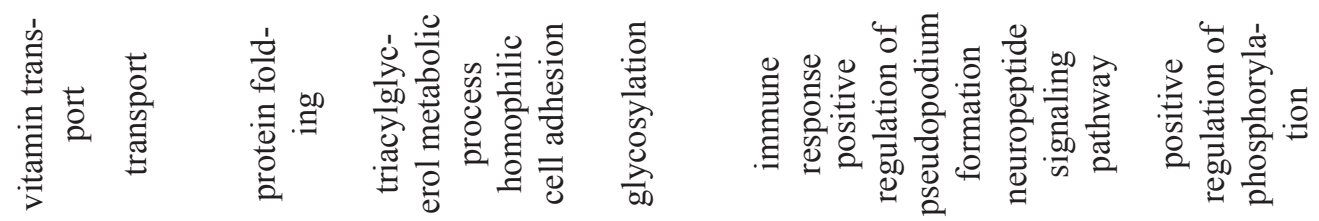

$\begin{array}{lllllllllll}8 & 8 & 8 & 8 & 8 & 8 & 0 & 0 & 0 & 0 & = \\ 0 & 0 & 0 & 0 & 0 & 0 & 0 & 0 & 0 & 0\end{array}$

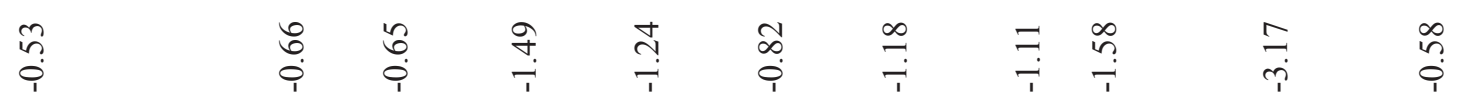

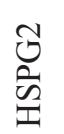

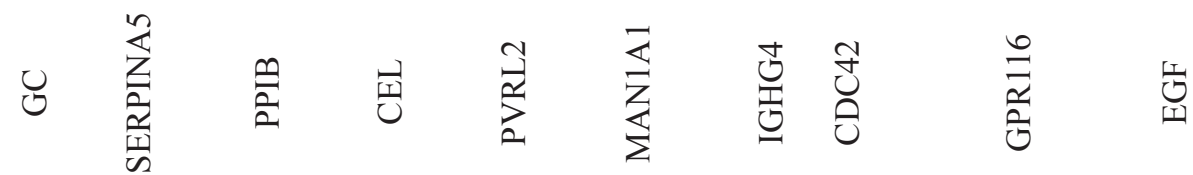

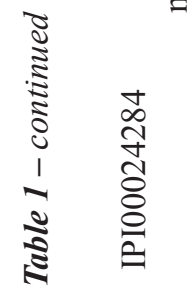

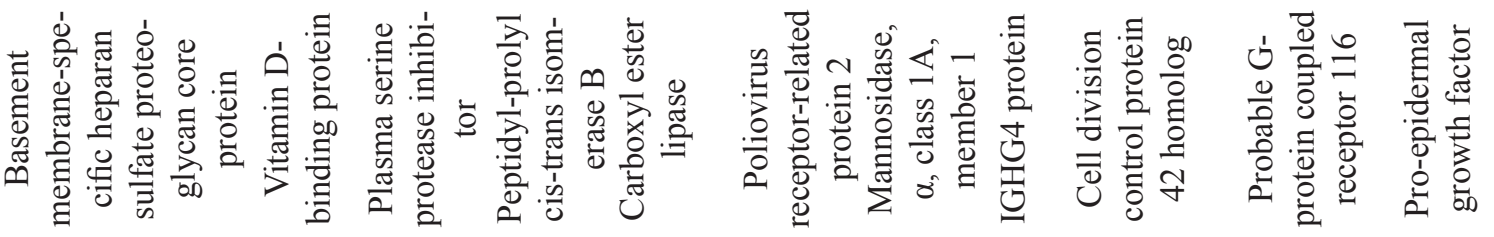

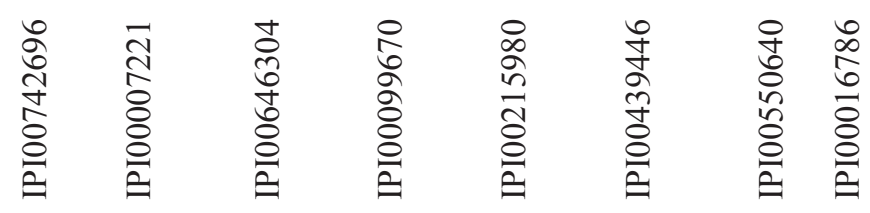

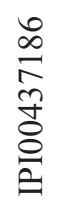

$\frac{n}{8}$ 
protein networks that may be involved in IC/PBS pathogenesis. A number of urine proteins were found to be differentially expressed between IC/PBS and control. Among the up-regulated proteins in IC/PBS, A1BG and ORM1 were present in all ten IC/PBS patients, with up-regulated expression in $\geq 60 \%$ of the cohort. TTR and HPX were found down-regulated in $\geq 60 \%$ of the IC/PBS cohort, and were present in all control urines. None of these four proteins have been previously implicated in IC/PBS pathogenesis. Two of these, A1BG and ORM1, are glycoproteins. Currently, many clinical biomarkers and therapeutic targets are glycoproteins, e.g. Her2/neu, PSA, and CA125.

165 and 193 proteins were found either in the IC/PBS or control urines, respectively. One interesting finding from these datasets was that pathway and network analyses identified possible activation of neurophysiological processes involved in synaptic inhibition, and lack of DNA damage repair in IC/PBS. One of the most important findings in pain research has been the identification of changes in the central nervous system (CNS), which may explain the perpetuation of pain in chronic pain syndromes (24). For example, in male chronic pelvic pain syndrome, responses to painful stimuli are changed, and evidence of nervous system alterations is present (25). CHL1 is known to be abundantly expressed in the CNS (e.g., brain, and spinal cord). Overall expression level of CHL1 was down-regulated in IC/PBS. Although no conclusions are being made as a result of these data, altered response to stimuli taken together with down-regulation of CNS proteins such as CHL1 may represent neurophysiological changes that contribute to IC/PBS pathogenesis.

Our strategy identified many differentially expressed proteins not previously associated with IC/PBS and this led to hypotheses around several novel network modules. These included glucose metabolism, alteration of which has been linked to human diseases (26). Natural killer cell mediated immune response has been well documented in various human diseases including prostate cancer (27). Little is known about involvement of glucose metabolism and natural killer cell immune response in IC/PBS. However, an association of glucose metabolism in IC/PBS has been recently detected by gene array analysis of experimentally induced IC in mice (28). Although the implication of these networks needs to be further investigated, any alteration to proteinprotein interactions could impact the natural cascade signaling process.

Although there is clear correspondence between pathological events and changes in protein expression in relevant networks and modules, whether any of the differentially expressed proteins are true markers for IC/PBS will require further investigation. In urine analysis, an individual's lifestyle, diet, medication history, and time of urine collection can influence the proteome profile; none of these factors were considered in urine collection in this or other studies. Another limitation of this study is the lack of detailed phenotyping of the subjects, which might aid data interpretation. However, this is a pilot study, and we were attempting to determine if our methods held merit for identifying selected proteins; furthermore, this small cohort would likely preclude any conclusions based on demographic or clinical variables.

\section{CONCLUSION}

Our preliminary data indicate that there are qualitative and quantitative differences between the urinary proteomes of women with and without IC/PBS. We identified a number of proteins as well as pathways/networks that might contribute to the pathology of IC/PBS or result from perturbations induced by this condition.

\section{ACKNOWLEDGMENT}

This work was supported by: National Institute of Diabetes and Digestive and Kidney Diseases U01 DK065202; National Institute of Environmental Health Sciences 5P30ES00703312; National Center For Research Resources 1S10RR023044-01, and Robert Wood Johnson Foundation 64189.

The authors thank Dr. Priska von Haller at the University of Washington South Lake Union Proteomics Resource for instrument support. 


\section{CONFLICT OF INTEREST}

\author{
None declared.
}

\section{REFERENCES}

1. Hanley RS, Stoffel JT, Zagha RM, Mourtzinos A, Bresette JF: Multimodal therapy for painful bladder syndrome / interstitial cystitis: pilot study combining behavioral, pharmacologic, and endoscopic therapies. Int Braz J Urol. 2009; 35: 467-74.

2. Hohlbrugger G, Lentsch P: Intravesical ions, osmolality and $\mathrm{pH}$ influence the volume pressure response in the normal rat bladder, and this is more pronounced after DMSO exposure. Eur Urol. 1985; 11: 127-30.

3. Wesselmann U: Neurogenic inflammation and chronic pelvic pain. World J Urol. 2001; 19: 180-5.

4. Anderson NL, Anderson NG: The human plasma proteome: history, character, and diagnostic prospects. Mol Cell Proteomics. 2002; 1(11): 845-67. Erratum in: Mol Cell Proteomics. 2003; 2: 50.

5. Adachi J, Kumar C, Zhang Y, Olsen JV, Mann M: The human urinary proteome contains more than 1500 proteins, including a large proportion of membrane proteins. Genome Biol. 2006; 7: R80.

6. Scherl A, Shaffer SA, Taylor GK, Kulasekara HD, Miller SI, Goodlett DR: Genome-specific gas-phase fractionation strategy for improved shotgun proteomic profiling of proteotypic peptides. Anal Chem. 2008; 80: 1182-91.

7. Goo YA, Liu AY, Ryu S, Shaffer SA, Malmström L, Page L, et al.: Identification of secreted glycoproteins of human prostate and bladder stromal cells by comparative quantitative proteomics. Prostate. 2009; 69: 49-61.

8. Liu H, Sadygov RG, Yates JR 3rd: A model for random sampling and estimation of relative protein abundance in shotgun proteomics. Anal Chem. 2004; 76: 4193-201.

9. Ashburner M, Ball CA, Blake JA, Botstein D, Butler H, Cherry JM, et al.: Gene ontology: tool for the unification of biology. The Gene Ontology Consortium. Nat Genet. 2000; 25: 25-9.

10. Tian M, Cui YZ, Song GH, Zong MJ, Zhou XY, Chen Y, et al.: Proteomic analysis identifies MMP-9, DJ-1 and $\mathrm{A} 1 \mathrm{BG}$ as overexpressed proteins in pancreatic juice from pancreatic ductal adenocarcinoma patients. BMC Cancer. 2008; 8: 241.

11. Narita T, Sasaki H, Hosoba M, Miura T, Yoshioka N, Morii T, et al.: Parallel increase in urinary excretion rates of immunoglobulin $\mathrm{G}$, ceruloplasmin, transferrin, and orosomucoid in normoalbuminuric type 2 diabetic patients. Diabetes Care. 2004; 27: 1176-81.

12. Altland K, Benson MD, Costello CE, Ferlini A, Hazenberg BP, Hund E, et al.: Genetic microheterogeneity of human transthyretin detected by IEF. Electrophoresis. 2007; 28: 2053-64.

13. Miller YI, Smith A, Morgan WT, Shaklai N: Role of hemopexin in protection of low-density lipoprotein against hemoglobin-induced oxidation. Biochemistry. 1996; 35: 13112-7.

14. Kneussel M: Dynamic regulation of GABA(A) receptors at synaptic sites. Brain Res Brain Res Rev. 2002; 39: 74-83.

15. Kanematsu T, Mizokami A, Watanabe K, Hirata M: Regulation of GABA(A)-receptor surface expression with special reference to the involvement of GABARAP (GABA(A) receptor-associated protein) and PRIP (phospholipase C-related, but catalytically inactive protein). J Pharmacol Sci. 2007; 104: 285-92.

16. Montag-Sallaz M, Baarke A, Montag D: Aberrant neuronal connectivity in CHL1-deficient mice is associated with altered information processing-related immediate early gene expression. J Neurobiol. 2003; 57: 67-80.

17. Dimitrakov J: A road map to biomarker discovery and validation in urological chronic pelvic pain syndrome. J Urol. 2008; 179: 1660-1.

18. Vera PL, Meyer-Siegler KL: Substance P induces localization of MIF/alpha1-inhibitor-3 complexes to umbrella cells via paracellular transit through the urothelium in the rat bladder. BMC Urol. 2006; 6: 24.

19. Zeng Y, Wu XX, Homma Y, Yoshimura N, Iwaki H, Kageyama S, et al.: Uroplakin III-delta4 messenger RNA as a promising marker to identify nonulcerative interstitial cystitis. J Urol. 2007; 178: 1322-7; discussion 1327.

20. Kim J, Keay SK, Freeman MR: Heparin-binding epidermal growth factor-like growth factor functionally antagonizes interstitial cystitis antiproliferative factor via mitogen-activated protein kinase pathway activation. BJU Int. 2009; 103: 541-6.

21. Keay SK, Zhang CO, Shoenfelt J, Erickson DR, Whitmore K, Warren JW, et al.: Sensitivity and specificity of antiproliferative factor, heparin-binding epidermal growth factor-like growth factor, and epidermal growth factor as urine markers for interstitial cystitis. Urology. 2001; 57(6 Suppl 1): 9-14.

22. Erickson DR, Tomaszewski JE, Kunselman AR, Stetter CM, Peters KM, Rovner ES, Demers LM, Wheeler MA, Keay SK: Urine markers do not predict biopsy findings or presence of bladder ulcers in interstitial 
cystitis/painful bladder syndrome. J Urol. 2008; 179: 1850-6.

23. Canter MP, Graham CA, Heit MH, Blackwell LS, Wilkey DW, Klein JB, et al.: Proteomic techniques identify urine proteins that differentiate patients with interstitial cystitis from asymptomatic control subjects. Am J Obstet Gynecol. 2008; 198: 553. e1-6.

24. Coderre TJ, Katz J, Vaccarino AL, Melzack R: Contribution of central neuroplasticity to pathological pain: review of clinical and experimental evidence. Pain. 1993; 52: 259-85.

25. Yang CC, Lee JC, Kromm BG, Ciol MA, Berger RE: Pain sensitization in male chronic pelvic pain syndrome: why are symptoms so difficult to treat? J Urol. 2003; 170: 823-6; discussion 826-7.
26. Holroyde CP, Gabuzda TG, Putnam RC, Paul P, Reichard GA: Altered glucose metabolism in metastatic carcinoma. Cancer Res. 1975; 35: 3710-4.

27. Suzuki K, Nakazato H, Matsui H, Hasumi M, Shibata Y, Ito K, et al.: NK cell-mediated anti-tumor immune response to human prostate cancer cell, PC-3: immunogene therapy using a highly secretable form of interleukin-15 gene transfer. J Leukoc Biol. 2001; 69: 531-7.

28. Tseng LH, Chen I, Chen MY, Lee CL, Lo TS, Lloyd LK: Genome-based expression profiles as a single standardized microarray platform for the diagnosis of experimental interstitial cystitis: an array of 75 genes model. Int Urogynecol J Pelvic Floor Dysfunct. 2009; 20. [Epub ahead of print]

Accepted after revision: March 15, 2010

\section{Correspondence address:}

Dr. Claire C. Yang

University of Washington, Department of Urology

Box 356510

Seattle, WA, 98195-6510, USA

Fax: + 1206 543-3272

E-mail:cyang@uw.edu

\section{EDITORIAL COMMENT}

I will say in order that biological meaning may be derived and testable hypotheses may be built from proteomic experiments in relation to IC/PBS, assignments of proteins detected by mass spectrometry must be supplemented with additional notation, such as information on known protein functions, protein-protein interactions, or biological pathway associations.

Visualizing this bulk of proteomic information and summarization the resulting significant differential expressed proteins underlying IC/PBC in an easy to navigate tabular formats, including meta-information on those proteins in addition to complementary gene ontology (GO) terminology, is also important so that in-house expertise on particular proteins may be integrated into the larger datasets.

Furthermore, proteins of interest underlying IC/PBC can be exported and matched to allow for re-searching of mass spectrometry data, and gene names corresponding to the proteins underlying IC/ PBS for further characterization, including pathway analysis. Therefore, I am hoping future published 
articles can make use of certain proteomic mapping and comparison tools (1-4) further investigating mass spectrometry and proteomic outputs in order to derive insight into the signaling pathway underlying IC/ PBS.

\section{REFERENCES}

1. Schmidt T, Frishman D: PROMPT: a protein mapping and comparison tool. BMC Bioinformatics. 2006; 7: 331 .
2. Gehlenborg N, O'Donoghue SI, Baliga NS, Goesmann A, Hibbs MA, Kitano H, et al.: Visualization of omics data for systems biology. Nat Methods. 2010; 7(3 Suppl): S56-68.

3. Yu K, Sabelli A, DeKeukelaere L, Park R, Sindi S, Gatsonis CA, et al.: Integrated platform for manual and high-throughput statistical validation of tandem mass spectra. Proteomics. 2009; 9: 3115-25.

4. Pruess M, Apweiler R: Bioinformatics Resources for In Silico Proteome Analysis. J Biomed Biotechnol. 2003; 2003: 231-236.

\section{Dr. Ling-Hong Tseng Department of Obstetrics and Gynecology Chang Gung Memorial Hospital Chang-Gung University College of Medicine Tao-Yuan, Taiwan E-mail:3g7330@yahoo.com.tw}

\section{EDITORIAL COMMENT}

Interstitial cystitis (IC) is a debilitating chronic disease caused by undetermined and unknown factors, which impedes the development of accurate diagnostic methods, therefore delaying the treatment of patients that otherwise would promptly be cared for. Currently, the diagnosis of IC in most cases is given by exclusion mainly because this pathology lacks adequate biologic markers in blood and urine.

Exact etiology of interstitial cystitis (IC) is unknown, however the impermeability of the urothelial barrier of the bladder just as an alteration in the production of urine proteins could play important physiological roles in lower urinary tract dysfunction (1).

Recently, the study of proteome has been introduced as a diagnostic tool for inflammatory diseases of difficult diagnoses. In diabetic nephropathy the proteomic marker can be a prognostic and/or a therapeutic factor (2).

In a preliminary study, the authors showed that there were qualitative and quantitative differences between the urinary proteomes of women with and without IC/PBS. Furthermore, future studies researching the proteome characteristics associated with IC could provide not only a better comprehension of physiopathology but also lead to further development of new drugs or therapies for treatment and/or prevention of IC and related disorders.

\section{REFERENCES}

1. Deng FM, Ding M, Lavker RM, Sun TT: Urothelial function reconsidered: a role in urinary protein secretion. Proc Natl Acad Sci U S A. 2001; 98: 154-9.

2. Thongboonkerd V: Current status of renal and urinary proteomics: ready for routine clinical application? Nephrol Dial Transplant. 2010; 25: 11-6.

\author{
Dr. João Luiz Amaro \\ Departamento de Urologia \\ Faculdade de Medicina de Botucatu \\ Botucatu, São Paulo, Brazil \\ E-mail:jamaro@fmb.unesp.br
}

